\title{
An In-silico Investigation of Natural Compounds Derived from Mimosa Pudica as Potential Therapeutic Candidate Against Mumps Virus
}

\author{
Jitendra Sasumana ${ }^{1}$ and Nillohit Mitra Ray ${ }^{2}$ \\ ${ }^{1}$ Department of Biotechnology, School of Bio-engineering and \\ Biosciences, Lovely Professional University, Punjab, India \\ ${ }^{2}$ Department of Biotechnology, School of Bioengineering \& Biosciences \\ Lovely Professional University, Phagwara, Punjab, India
}

\section{ABSTRACT}

Mumps is a viral disease, which causes respiratory illness in humans. The symptoms involve swelling of one or both the parotid salivary glands. Among all the viral surface proteins in Mumps, the hemagglutinin-neuraminidase, MuV$\mathrm{HN}$, has a vital role in the entry of virus into the host cells and causing infection. Therefore, MuV-HN can be used as an ideal target in designing of novel inhibitors against the virus. In a drug designing approach, the protein-ligand interaction is one of the major aspect which gives potential candidates serving as an inhibitor against the mumps virus Hemagglutinin- neuraminidase protein. By performing docking studies, a detailed analysis of the protein-ligand interaction of the active compounds of plant Mimosa pudica against the MuV-HN protein was reported. The results confirmed that for each ligand there is a consistent binding affinity with specific energy. Among all the active compounds of Mimosa pudica, riboflavin and sitosterol showed efficient docking score which are $-9 \mathrm{kcal} / \mathrm{mol}$ and $-10.7 \mathrm{kcal} / \mathrm{mol}$ respectively against the MuV-HN. These results depict that out of the different phytocompounds of Mimosa pudica, riboflavin and sitosterol can be used as efficient candidates in pharmacokinetic and pharmacodynamic studies in developing a novel inhibitor against Mumps virus.

KEY WORDS: MUMPS, INHIBITORS, MUV-HN, DOCKING, MIMOSA PUDICA.

\section{INTRODUCTION}

Mumps is a viral disease which involves the painful swelling of one or both the parotid salivary glands (Hviid et al., 2008). It has been known to mankind since antiquity and has been mentioned by Hippocrates as early as $400 \mathrm{BC}$. It was first described scientifically by a

\section{ARTICLE INFORMATION}

Received 10th Oct 2020 Accepted after revision 25th Dec 2020 Print ISSN: 0974-6455 Online ISSN: 2321-4007 CODEN: BBRCBA

Thomson Reuters ISI Web of Science Clarivate Analytics USA and Crossref Indexed Journal

\section{Clarivate
Analytics}

NAAS Journal Score 2020 (4.31)

A Society of Science and Nature Publication,

Bhopal India 2020. All rights reserved.

Online Contents Available at: http//www.bbrc.in/

Doi: $h t t p: / / d x$.doi.org/10.21786/bbrc/13.15/59
British physicist Robert Hamilton in 1790. However, its highly contagious nature was not proved until 1934 by C.D. Johnson and E.W. Good pasture. Prior to its vaccine development, it had significantly affected a sizeable portion of the human population, which also involved the armies of both the world wars (Sama et al., 2011).

It is a highly contagious disease spread by respiratory droplets from an infected person or by direct contact. Before the development of a vaccine, it was a common childhood disease worldwide with a large scale outbreak occurring every 2-5 years (Hviid et al., 2008). Early symptoms include fever, migraine, poor craving and feeling commonly unwell. These symptoms, however appear after 16-18 days of incubation period after contact with the virus. This is then followed by the swelling of parotid glands, which is the most obvious indicator of 
mumps disease. The afflicted person becomes contagious about 7 days before onset of parotid gland swelling to about 8 days after this onset. About 20-40\% of the infected individuals do not show the early symptoms, and hence spread the infection without knowing. Once the disease has run its course, the person becomes typically immunized for life, i.e. does not have the disease again (Atkinson et al., 2006) (Kutty et al., 2010).

Prior to development of a vaccine, typically $0.1-1 \%$ of the world population was affected annually with mumps. Vaccine development has resulted in a decline of more than 90\% of this disease (Hviid et al., 2008). However, it remains a problem in the developing nations where vaccination is less common (Farrar et al., 2013). Instances of co-infection with other disease have also occurred. For example, in about 25\% of the mumps cases, occurrence of mumps-induced viral encephalitis has been reported. Prior to vaccine development mumps was the fundamental driver of viral encephalitis in numerous nations. Contracting the disease during the initial 12 weeks of pregnancy is related with a 25\% frequency of unconstrained premature birth. Also, in children, acquired sensoryneural deafness is mainly caused by mumps (Pietrantonj et al., 2020).

There is no particular treatment for the mumps. Drugs such as paracetamol are administered for controlling the pain due to side effects. In specific cases, intravenous immunoglobulin administration is carried out. In case of co-infection with meningitis or pancreatitis, hospitalization may be required (Hviid et al., 2008). Recently there has been renewed focus to study the antigenic diversity of mumps virus due to occasional resurgence of mumps epidemics. The mumps virus is an enveloped, single-stranded, linear negative-sense RNA virus whose genome consists of 15,384 bases encoding nine proteins (Hviid et al., 2008). Various bioniformatical approaches have been applied to study the mumps virus; docking being the most popular one.

Docking Studies Previously Carried Out With Mumps Virus: Very few docking studies have been performed on mumps virus. In these studies, it has been mentioned that Mumps Virus (MuV) Hemagglutinin-Neuraminidase (HN), which is one the viral surface glycoproteins, has a vital role in entry of virus into host cells. This causes infection and hence this viral glycoprotein represents an ideal target for design of novel inhibitor. Hence the structural basis of docking studies involves glycan-receptor binding by $\mathrm{HN}$ glycoprotein of mumps virus. Analysis of the molecular recognition of the silylatedglycans of the host cell surface by MuV-HN protein been reported in these docking studies. The structural features of sialoglycans and MuV-HN have been revealed by using NMR, docking, molecular modelling and CORCEMA-ST (Complete Relaxation and Conformational Exchange Matrix Analysis of Saturation Transfer) software. These reports provided the roots for design of structure based drugs against mumps-induced diseases (Forgione et al., 2020).
Figure 1: Close up view of ligand 1 binding mode at the MuV-HN active site, drawn by using Pymol 2.3 Software (https://pymol.org/2/). The main amino acid residues involved in the binding are represented in stick. Galactose and $\mathrm{N}$-acetyl Glucosamine residues are depicted in yellow and blue, respectively (Forgione et al., 2020).

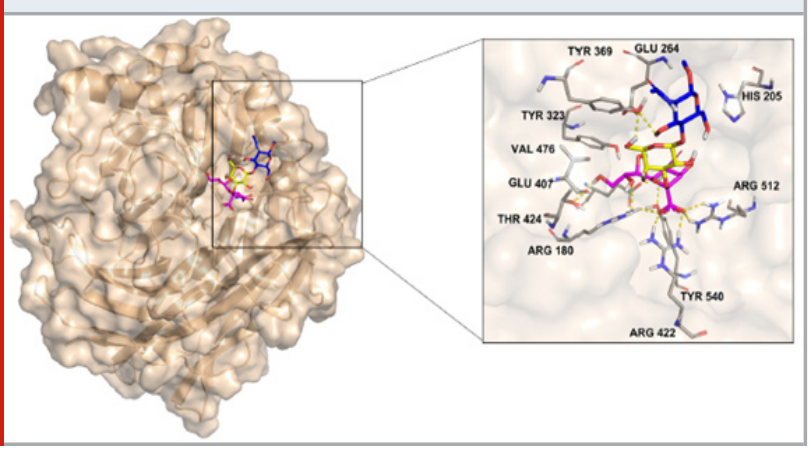

Forgione et al. performed a detailed study on the structural basis of glycan-receptor binding of MuV-HN. They retrieved the monomeric structure of $\mathrm{MuV}-\mathrm{HN}$ receptor binding head space (PDB ID, 5b2c) and utilized it for docking computations (Fig 1). The docking of MuV-HN structure was donewith 3' sialyllactose ligand (PDB-ID: 5b2d).The 3D structure of $3^{\prime}$ sialyllactose was separated from the PDB document.Ligands were readied for docking estimations utilizing AutoDockTools, liberating all rotatable bonds to move, aside from the glycosidic linkages, during the docking computations. Missing hydrogen iotas were included, and protonation condition of ionizable bunches was registered by utilizing Maestro Protein Preparation Wizard. The structure was submitted to 100000 stages of steepest drop minimization with MacroModel and upgradedwith OPLS_2005 power field. Docking computations of all mixes were performed by utilizing AutoDock 4.2.2 (Forgione et al., 2020).

Figure 2: Interaction model V-STAT1-STAT2. Heterodimer model of STAT1-STAT2 by the SH2-domain (STAT1 PDB: 1YVL and 3D model of STAT2 from 1YVL and 1BF5). Heterodimer model shows the following residues: Tyr690 and Tyr701 (orange), nuclear localization signal residues (pink), lysine residues to ubiquitylation (Ub) (blue). Interaction of STATs heterodimer with VWT by Trp-motif and STAT2. Interaction of heterodimer with VGly by STAT2, lysine residues (Ub) (blue) (Murrieta et al., 2010).

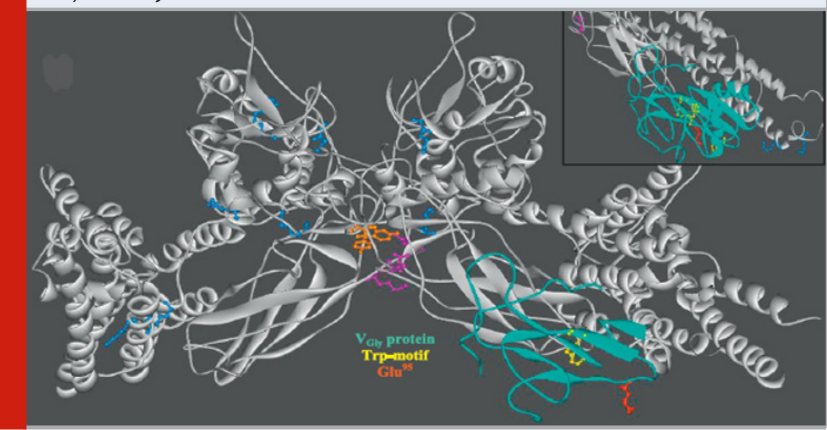


Murrieta et al. studied the interaction of Urabe AM9 strain of mumps virus $\mathrm{V}$ protein with STAT1-STAT2 homodimer protein complex of cell signaling pathway in humans. The $\mathrm{V}$ protein has the ability to inhibit the interferon-mediated antiviral response by inducing the degradation of STAT proteins (Murrieta et al., 2010).

Table 1 . The compounds of Mimosa pudica used in the docking

Compounds of Mimosa pudica

\begin{tabular}{|l|c|c|}
\hline Ascorbic-Acid & Linoleic-Acid & Palmitic-Acid \\
\hline Beta-Carotene & Linolenic-Acid & Riboflavin \\
\hline Crocetin & Mimosine & Sitosterol \\
\hline D-Glucuronic-Acid & Norepinephrine & Stearic-Acid \\
\hline D-Xylose & Oleic-Acid & Thiamin \\
\hline
\end{tabular}

Hypothetical heterodimer STAT1-STAT2 model was acquired by a docking examination with Hex worker. The putative connection models among VWT and VGly with STAT1-STAT2 proteins were created with PatchDock worker (Molecular Docking Algorithm Based on Shape Complementary Principles) and 1000 hypothetical models were refined on FireDock (Fast Interaction Refinement in Molecular Docking) (Fig 2). Kubota et al. studied the interaction of mumps virus $\mathrm{V}$ protein with receptor of activated protein $\mathrm{C}$ kinase 1(RACK1). This interaction results in dissociation of STAT- 1 from the Alpha Interferon Receptor Complex. It has been stated that the C-terminal Cys-rich locale of mumps V protein (Vsp) is related with obstructing of the interferon (IFN) signal transduction pathway through an abatement in STAT-1 creation.

Table 2. Docking scores after docking the ligands with MuV-HN protein

\begin{tabular}{|l|c|c|c|c|c|}
\hline Ligand & Affınity (kcal/mol) & Ligand & Affinity (kcal/mol) & Ligand & Affinity (kcal/mol) \\
\hline Ascorbic-Acid & -5.8 & Linoleic-Acid & -5.4 & Palmitic-Acid & -5.8 \\
\hline Beta-Carotene & -7.2 & Linolenic-Acid & -5.8 & Riboflavin & -9 \\
\hline Crocetin & -6.7 & Mimosine & -5.9 & Sitosterol & -10.7 \\
\hline D-Glucuronic-Acid & -6.2 & Norepinephrine & -5.9 & Stearic-Acid & -5.7 \\
\hline D-Xylose & -5.7 & Oleic-Acid & -5.7 & Thiamin & -7.5 \\
\hline
\end{tabular}

The intracellular objective of the $\mathrm{V}$ protein was researched by utilizing a two-mixture screening framework with Vsp as lure. Full-length V protein and Vsp had the option to tie to RACK1; the cooperation didn't need two WD spaces, WD1 and WD2, in RACK1. A critical association between $\mathrm{V}$ protein and RACK1 was likewise exhibited in cells steadily contaminated with mumps infection, and the arrangement of the complex was not influenced by treatment with IFN (Kubota et al., 2002).

Docking Studies Carried Out in this Work: Our docking studies involve hemagglutinin-neuraminidase, which is one the viral surface glycoproteins and has a vital role in entry of mumps virus into host cells. This viral glycoprotein is an ideal target for design of novel inhibitor (Forgione et al., 2020). As the extracts of Indian medicinal plant Mimosa pudica has the ability to induce the anti-viral activity against the mumps virus, we performed the docking of hemagglutinin-neuraminidase protein of mumps virus with the several compounds of Mimosa pudica. The active compounds were retrieved from the species of plant Mimosa Pudica (refer Table 1) (Malayan et al., 2013).

\section{MATERIAL AND METHODS}

For molecular docking studies, the structures of ligand and protein have to be retrieved and refined. The most prominent and active compounds (ligands) (Table 1) present in Mimosa Pudica were subjected to molecular docking analysis against the MuV-HN protein that currently serves as a promising target for effective drug development against mumps virus. The $\mathrm{X}$-ray diffraction crystal structure of the MuV-HN head domain in the monomeric form was retrieved from PDB (PDB ID: 5b2c). The water molecules were removed from the PDB file. Then, using AutoDock tools, polar hydrogen atoms and Kollman charges were added. The structure of the phytocompounds (Table 1) were retrieved from PubChem and their respective three dimensional structures were generated through open Babel software (0'Boyle et al., 2011). Then energy optimization and minimization of these structures was performed using PRODRG server (Schüttelkopf et al., 2004). Lastly, using AutoDock tools, Gasteiger charges were added to the corresponding ligand structures.

Molecular docking was performed using the AutoDockVina software (Naidoo et al., 2004) (Trott et al., 2010). The ligands were docked to the active region of the MuV-HN protein using a grid based docking method involving a rigid protein receptor and flexible ligand docking protocol. The grid size was set at 100,100, 100 and the center was set at 90.514, 154.133, -28.032. The ligandreceptor complexes with lowest binding scores were analyzed for studying the interactions using the PyMol software (Naidoo et al., 2004). The ligand-receptor binding interaction results are given in Table 2. 
Figure 3: The interaction of riboflavin and sitosterol with hemagglutinin-neuraminidase. A. The interaction of riboflavin. B. The interaction of sitosterol. C. The hydrophobic interaction and hydrogen bonding of sitosterol. D. The hydrophobic interaction (Brown dashed lines) and Hydrogen bonding (Yellow dashed lines) of riboflavin.

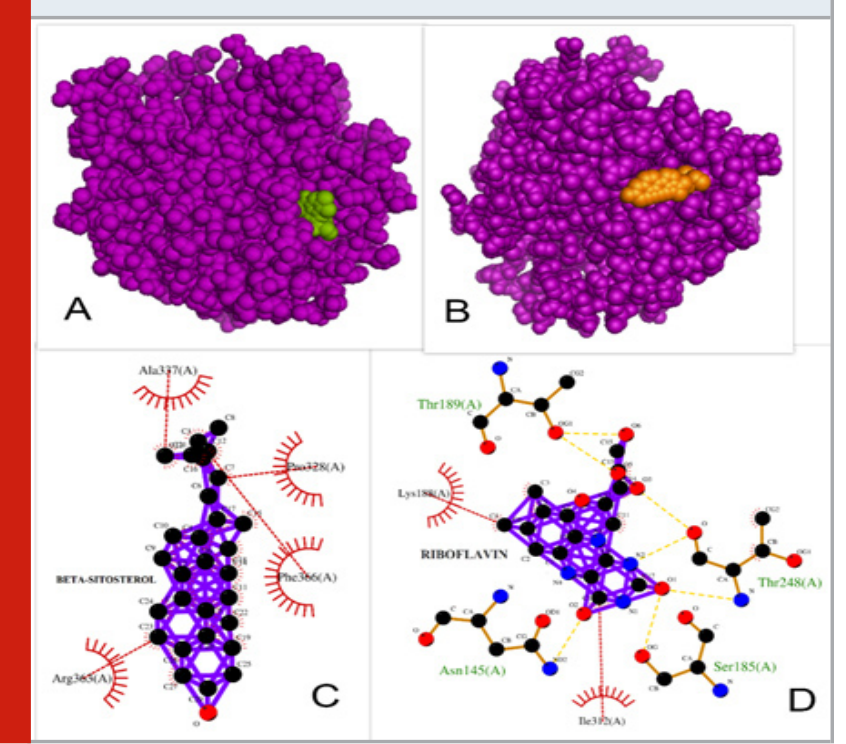

RESULTS AND DISCUSSION

The docking analysis showed that for each ligand a consistent binding affinity with specific energy was obtained (refer Table 2). Out of all the ligands riboflavin and sitosterol had the good docking score against MuV-HN (highlighted in yellow, Table 2). These two interactions are shown in Figure 3. Since they have a binding affinity score of less than $-7 \mathrm{Kcal} / \mathrm{mol}$, they can be used as potential candidates for further pharmacokinetic and pharmacodynamics studies in wet lab against the $\mathrm{MuV}-\mathrm{HN}$ protein. The hydrophobic interactions as well as hydrogen bonding between these ligands and MuV$\mathrm{HN}$ receptor are depicted in figure 3.

\section{CONCLUSION}

A drug designing approach based on protein- ligand interaction was used to find out potential candidates which could serve as an inhibitor against the mumps virus Hemagglutinin- neuraminidase protein. Various phytocompounds derived from the plant Mimosa Pudica were analysed as ligands for this purpose. The in-silico studies uncoveredpositive outcomes for use of these compounds as potential inhibitors of MuV-HN. 15 phytocompounds from Mimosa Pudica were used to carry out docking studies with MuV-HN. Out of these, two compounds, namely sitosterol and riboflavin showed high binding affinity towards the pathogenic glycoprotein. The information acquired from thus study can accelerate the development of novel medication against mumps virus which would be a boon for developing nations where mumps outbreaks still occur. The data obtained through this work has to be further verified by doing in-vitro and in vivo studies for the deveopment of novel inhibitors against mumps virus.

\section{REFERENCES}

Atkinson, W., (2006). Epidemiology and prevention of vaccine-preventable diseases. Department of Health \& Human Services, Centers for Disease Control and Prevention.

Di Pietrantonj, C., Rivetti, A., Marchione, P., Debalini, M.G. and Demicheli, V., (2020). Vaccines for measles, mumps, rubella, and varicella in children. Cochrane Database of Systematic Reviews, (4).

Farrar, J., Hotez, P.J., Junghanss, T., Kang, G., Lalloo, D. and White, N.J., (2013). Manson's Tropical Diseases E-Book. Elsevier Health Sciences.

Forgione, R.E., Di Carluccio, C., Kubota, M., Manabe, Y., Fukase, K., Molinaro, A., Hashiguchi, T., Marchetti, R. and Silipo, A., (2020). Structural basis for Glycanreceptor binding by mumps virus hemagglutininneuraminidase. Scientific reports, 10(1), pp.1-13.

Hviid, A., Rubin, S. and Mühlemann, K., (2008). Mumps. The Lancet, 371(9616), pp.932-944.

Kubota, T., Yokosawa, N., Yokota, S.I. and Fujii, N., (2002). Association of mumps virus V protein with RACK1 results in dissociation of STAT-1 from the alpha interferon receptor complex. Journal of virology, 76(24), pp.12676-12682.

Kutty, P.K., Kyaw, M.H., Dayan, G.H., Brady, M.T., Bocchini Jr, J.A., Reef, S.E., Bellini, W.J. and Seward, J.F., 2010. Guidance for isolation precautions for mumps in the United States: a review of the scientific basis for policy change. Clinical infectious diseases, 50(12), pp.1619-1628.

Malayan, J., Selvaraj, B., Warrier, A., Shanmugam, S., Mathayan, M. and Menon, T., (2013). Anti-mumps virus activity by extracts of Mimosa pudica, a unique Indian medicinal plant. Indian Journal of Virology, 24(2), pp.166-173.

Naidoo, D., Roy, A., Slav囚tínská, L.P., Chukwujekwu, J.C., Gupta, S. and Van Staden, J., (2020). New role for crinamine as a potent, safe and selective inhibitor of human monoamine oxidase B: In vitro and in silico pharmacology and modeling. Journal of Ethnopharmacology, 248, p.112305.

O’Boyle, N.M., Banck, M., James, C.A., Morley, C., Vandermeersch, T. and Hutchison, G.R., (2011). Open Babel: An open chemical toolbox. Journal of cheminformatics, 3(1), p.33.

Pinkbook | Mumps | Epidemiology of Vaccine Preventable Diseases | CDC". www.cdc.gov. 25 September 2019. Archived from the original on 22 April 2017.

Rosas-Murrieta, N.H., Herrera-Camacho, I., Palma- 
Ocampo, H., Santos-López, G. and Reyes-Leyva, J., (2010). Interaction of mumps virus V protein variants with STAT1-STAT2 heterodimer: experimental and theoretical studies. Virology journal, 7(1), pp.1-10.

Samal, S.K. ed., (2011). The biology of paramyxoviruses. Horizon Scientific Press. .

Schüttelkopf, A.W. and Van Aalten, D.M., (2004). PRODRG: a tool for high-throughput crystallography of protein-ligand complexes. Acta Crystallographica Section D: Biological Crystallography, 60(8), pp.13551363.

Trott, 0. and Olson, A.J., (2010). Auto Dock Vina: improving the speed and accuracy of docking with a new scoring function, efficient optimization, and multithreading. Journal of computational chemistry, 31(2), pp.455-461. 